\title{
Enantioselective Synthesis of (2R,3R,7S)-3,7 -Dimethylpentadecan-2-ol, Sex Pheromone Component of Pine Sawflies
}

\author{
Jardel A. Moreira and Arlene G. Corrêa* \\ Departamento de Química, Universidade Federal de São Carlos, 13565-905, São Carlos - SP, Brazil
}

\begin{abstract}
A mosca Neodiprion sertifer (Geoffrey) (Hymenoptera: Diprionidae) é uma importante praga em florestas de pinheiro no norte da Europa, Ásia e América do Norte. Os componentes majoritários do feromônio sexual desta espécie são acetatos ou propionatos do 3,7-dimetilpentadecan-2-ol (diprionol). Neste trabalho relatamos a síntese enantiosseletiva do $(2 R, 3 R, 7 S)$-diprionol em 12 etapas ( $7.5 \%$ de rendimento total), partindo-se do (-)-isopulegol comercial.
\end{abstract}

The pine sawfly, Neodiprion sertifer (Geoffrey) (Hymenoptera: Diprionidae), is a widespread and economically important forest insect in North America, Japan and Europe. It has been demonstrated that the main sex pheromone components of this species are acetate or propionate of 3,7-dimethyl-2-pentadecanol (diprionol). We describe herein an enantioselective synthesis of $(2 R, 3 R, 7 S)$-diprionol in 12 steps ( $7.5 \%$ overall yield), starting from commercial (-)-isopulegol.

Keywords: 3,7-dimethylpentadecan-2-ol, diprionol, Neodiprion sertifer, sex pheromone

\section{Introduction}

Insect pest management, monitoring and control programs utilizing sex pheromones as behavior modifying chemicals have become important for several insect groups, particularly moths. Such programs frequently include masstrapping and mating disruption techniques. This development has been accompanied by the search for simple, efficient and less aggressive synthetic methodologies for the preparation of pheromones.

The pine sawfly, Neodiprion sertifer (Geoffrey) (Hymenoptera: Diprionidae), is a widespread and economically important forest insect in North America, Japan and Europe. Field tests and electrophysiological experiments demonstrated that males of several Neodiprion species are strongly attracted by diprionyl acetate, while some species of this genus, and of the genus Diprion, prefer the corresponding propionate. It has been suggested that species specificity in diprionid sawflies is based on different combinations of the isomers of diprionyl acetate and/or propionate ${ }^{1}$.

Anderbrant et al. have investigated the geographic pattern of male sawfly response at eight field sites ranging from Japan in the east to Canada in the west ${ }^{2}$. They found an increased inhibitory effect of the $(2 S, 3 R, 7 R)$-isomer from Japan and Siberia to Europe. At the eastern sites, increasing

*e-mail: agcorrea@dq.ufscar.br amounts of the $(2 S, 3 R, 7 R)$-isomer up to and equal to the amount of the $(2 S, 3 S, 7 S)$-isomer, did not influence the trap catch, whereas at sites in Europe, as little as $1 \%$ of the $(2 S, 3 R, 7 R)$-isomer almost completely inhibited the attraction. The response of the North American population was intermediate. The only site in which the $(2 S, 3 R, 7 R)$ isomer was essential for the attraction of males was in Siberia. A similar pattern was found for the $(2 S, 3 R, 7 S)$-isomer. Both the acetate and the propionate form of the $(2 S, 3 S, 7 S)$-isomer were attractive by themselves in Japan, Europe and North America, and neither the $(2 S, 3 R, 7 S)$-isomer nor the $(2 S, 3 R, 7 R)$-isomer alone were attractive, in the acetate or propionate form.

It is evident from the facts discussed above that diprionyl esters with high degree of enantiomeric purity and well-known composition are necessary to accomplish reliable biological tests or ultimately for field application. Several research groups have synthesized racemic ${ }^{3}$ or optically enriched stereoisomers of diprionol and its esters $^{4,5}$. Högberg et al. have prepared all eight diprionol isomers using a convergent approach in 22 steps. The synthetic intermediates were prepared from tartaric acid and by asymmetric synthesis using either an oxazoline or a prolinol derivative as chiral auxiliary ${ }^{6}$.

In this paper we present the enantioselective synthesis of $(2 R, 3 R, 7 S)$-diprionol from commercially available (-)isopulegol in 12 steps. Our synthetic strategy was based on 
a methodology developed by Ferreira and Zarbin ${ }^{7}$ and applied for the synthesis of two isomers of methyl 2,6,10trimethyldodecanoate, male-produced pheromone of stink bugs Euschistus spp.

\section{Experimental}

Unless otherwise noted, all commercially available reagents were purchased from Aldrich Chemical Co. Reagents and solvents were purified when necessary according to the usual procedures described in the literature. The IR spectra refer to films and were measured on a Bomem M102 spectrometer. ${ }^{1} \mathrm{H}$ and ${ }^{13} \mathrm{C}$ NMR spectra were recorded on a Bruker ARX-200 (200 and $50 \mathrm{MHz}$ respectively) and DRX-400 (400 and $100 \mathrm{MHz}$ respectively) spectrometers. Optical rotations were measured on a Perkin-Elmer 241 polarimeter. Mass spectra were recorded on a Shimadzu GCMS-QP5000. Analytical thin-layer chromatography was performed on a $0.25 \mu \mathrm{m}$ film of silica gel containing fluorescent indicator $\mathrm{UV}_{254}$ supported on an aluminum sheet (Sigma-Aldrich). Flash column chromatography was performed using silica gel (Kieselgel 60, 230-400 mesh, E. Merck). Gas chromatography was performed in a Shimadzu GC-17A with $\mathrm{H}_{2}$ as carrier and using a DB-5 column. Chiral GC was performed in a HP 5890 with $\mathrm{H}_{2}$ as carrier (14 psi) and using an heptakis (2,6-di- $O$-methyl-3- $O$-phenyl)- $\beta$ cyclodextrin column $(20 \%$ in OV 1701 , w/w, $25 \mathrm{~m}, 0,25 \mathrm{~mm}$ i.d.) column. Elemental analyses were performed on a Fisons EA 1108 CHNS-O.

\section{(-)-(1R,3R,4S,8R)-p-Menthane-3,9-diol (3)}

$\mathrm{BF}_{3} \cdot \mathrm{Et}_{2} \mathrm{O}\left(2.8 \mathrm{~cm}^{3}, 22 \mathrm{mmol}\right)$ was added dropwise to a stirred suspension of $\mathrm{NaBH}_{4}(0.740 \mathrm{~g}, 19.6 \mathrm{mmol})$ in diglyme $\left(13 \mathrm{~cm}^{3}\right)$ at room temperature under nitrogen. The generated diborane was transferred via a cannula into a solution of (-)isopulegol $(2.00 \mathrm{~g}, 13.0 \mathrm{mmol})$ in dry THF $\left(52 \mathrm{~cm}^{3}\right)$ under nitrogen at $0^{\circ} \mathrm{C}$. After stirring for $3 \mathrm{~h}, \mathrm{H}_{2} \mathrm{O}\left(2.0 \mathrm{~cm}^{3}\right)$ was slowly added, followed by $\mathrm{H}_{2} \mathrm{O}_{2}$ (30 wt. \% solution in water, $\left.3.0 \mathrm{~cm}^{3}\right)$ and aqueous solution of $\mathrm{NaOH}\left(30 \%, 3.0 \mathrm{~cm}^{3}\right)$, and stirred for an additional $30 \mathrm{~min}$ at room temperature. The reaction mixture was extracted with ether $\left(3 \times 40 \mathrm{~cm}^{3}\right)$ and the separated organic layer was washed with brine (30 $\mathrm{cm}^{3}$ ) and dried over $\mathrm{MgSO}_{4}$. The oil obtained was purified by column chromatography (hexane: ethyl acetate, 1:2 v/ $\mathrm{v}$ ), followed by recrystallization (cyclohexane: $\mathrm{CH}_{2} \mathrm{Cl}_{2}$, $2: 1 \mathrm{v} / \mathrm{v})$ to afford diol $\mathbf{3}(1.88 \mathrm{~g}, 84 \%$ yield $)$ as colorless crystals. $[\alpha]_{\mathrm{D}}{ }^{25}-18.5\left(\right.$ c $\left.5.00, \mathrm{CHCl}_{3}\right)$, Lit. $^{7}:[\alpha]_{\mathrm{D}}{ }^{25}-18.6$ (c 10.0, $\mathrm{CHCl}_{3}$ ); IR $v_{\max } / \mathrm{cm}^{-1} 3234,2961,2945,2912$, 1451, 1037 (film); ${ }^{1} \mathrm{H}$ NMR (400 MHz, $\mathrm{CDCl}_{3}$ ) $\delta 0.92$ (d, $J 6.5 \mathrm{~Hz}, 3 \mathrm{H}), 0.96(\mathrm{~d}, J 7.3 \mathrm{~Hz}, 3 \mathrm{H}), 1.24(\mathrm{dq}, J 3.5$ and 12.8 $\mathrm{Hz}, 1 \mathrm{H}), 1.35$ (ddd, $J 2.0,3.2$ and $9.8 \mathrm{~Hz}, 1 \mathrm{H}), 1.38-1.46$ (m,
$1 \mathrm{H}), 1.56(\mathrm{dq}, J 3.2$ and $13.0 \mathrm{~Hz}, 1 \mathrm{H}), 1.64$ (dquin, $J 3.0$ and $12.5 \mathrm{~Hz}, 1 \mathrm{H}$ ), 1.78 (br s, 1H), 1.80-1.88 (m, 1H), 1.96 (ddd, $J$ $1.8,3.9$ and $12.1 \mathrm{~Hz}, 1 \mathrm{H}), 3.36(\mathrm{br} \mathrm{s}, 1 \mathrm{H}), 3.45(\mathrm{td}, J 4.3$ and $10.6 \mathrm{~Hz}, 1 \mathrm{H}), 3.56(\mathrm{dd}, J 3.4$ and $10.7 \mathrm{~Hz}, 1 \mathrm{H}), 3.65(\mathrm{dd}, J$ 5.5 and $10.7 \mathrm{~Hz}, 1 \mathrm{H}) ;{ }^{13} \mathrm{C} \mathrm{NMR}\left(100 \mathrm{MHz}, \mathrm{CDCl}_{3}\right) \delta 11.9$, 22.1, 29.5, 31.4, 34.6, 38.6, 44.6, 48.5, 67.2, 70.2; GC-MS $(70 \mathrm{eV}) \mathrm{m} / \mathrm{z} .172\left(\mathrm{M}^{+}, 0.6\right), 154$ (4.8), 139 (8.8), 112 (19.9), 95 (36.6), 81 (96.4), 71 (42.8), 55 (100).

\section{(-)-(1R,3R,4S,8R)-9-Benzyloxy-p-menthan-3-ol (4)}

Diol 3 (1.60 g, $9.3 \mathrm{mmol}$ ) was added dropwise to a stirred suspension of $\mathrm{NaH}(60 \%$ dispersion in mineral oil, $0.41 \mathrm{~g}, 10.2 \mathrm{mmol})$ in dry $\operatorname{DMF}\left(32 \mathrm{~cm}^{3}\right)$ under $\mathrm{N}_{2}$ at $0{ }^{\circ} \mathrm{C}$. The resulting solution was cooled at $-10^{\circ} \mathrm{C}$ and stirred for $1.5 \mathrm{~h}$. The mixture was cooled at $-60^{\circ} \mathrm{C}$ and a solution of benzyl bromide $\left(1.10 \mathrm{~cm}^{3}, 9.3 \mathrm{mmol}\right)$ in DMF $\left(6.0 \mathrm{~cm}^{3}\right)$ was slowly added. The mixture was stirred at $-60^{\circ} \mathrm{C}$ for 2 $\mathrm{h}$, then the reaction temperature was raised to room temperature, and stirred for an additional $12 \mathrm{~h}$. Saturated aqueous $\mathrm{NH}_{4} \mathrm{Cl}\left(30 \mathrm{~cm}^{3}\right)$ was added, and the resulting mixture was extracted with ethyl acetate $\left(3 \times 40 \mathrm{~cm}^{3}\right)$. The organic layer was washed with brine $\left(30 \mathrm{~cm}^{3}\right)$ and dried over $\mathrm{Na}_{2} \mathrm{SO}_{4}$, concentrated and the oil obtained was purified by column chromatography (hexane: ethyl acetate, $5: 1 \mathrm{v} / \mathrm{v}$ ) to afford compound 4 (2.01 g, 83\% yield) as a colorless oil. $[\alpha]_{\mathrm{D}}{ }^{25}-13.1\left(c 1.88, \mathrm{CHCl}_{3}\right)$, Lit. ${ }^{7}:[\alpha]_{\mathrm{D}}{ }^{25}-12.4(c$ 14.6, $\left.\mathrm{CHCl}_{3}\right)$ ]; IR $v_{\text {max. }} / \mathrm{cm}^{-1} 3423,3963,3030,2940$, 2919, 1454, 1096 (film); ${ }^{1} \mathrm{H}$ NMR (200 MHz, $\mathrm{CDCl}_{3}$ ) $\delta$ 0.84-0.89 (m, 1H), 0.91 (d, J 6.4 Hz, 3H), 0.96 (d, J 7.4 Hz, $3 \mathrm{H}), 1.14(\mathrm{dd}, J 3.4$ and $12 \mathrm{~Hz}, 1 \mathrm{H}), 1.22-1.80(\mathrm{~m}, 5 \mathrm{H})$, $1.85-2.15(\mathrm{~m}, 2 \mathrm{H}), 3.36-3.55(\mathrm{~m}, 3 \mathrm{H}), 3.62$ (br s, $1 \mathrm{H}), 4.48$ $(\mathrm{d}, J 12.0 \mathrm{~Hz}, 1 \mathrm{H}), 4.56(\mathrm{~d}, J 12.0 \mathrm{~Hz}, 1 \mathrm{H}), 7.26-7.55(\mathrm{~m}$, $5 \mathrm{H}) ;{ }^{13} \mathrm{C} \mathrm{NMR}\left(50 \mathrm{MHz}, \mathrm{CDCl}_{3}\right) \delta 13.6,22.2,27.9,31.5$, 34.7, 35.5, 44.0, 49.0, 70.5, 73.4, 74.4, 127.7 (3C), 128.5 (2C), 137.8; GC-MS (70 eV) m/z 171 (2.7), 153 (15.5), 138 (14.5), 123 (6.6), 107 (14.3), 95 (30.2), 91 (100), 81 (34.3), 55 (34.3), 41 (44.1).

\section{(-)-(1R,4S,8R)-9-Benzyloxy-p-menthan-3-one (5)}

Alcohol 4 (3.38 g, $12.9 \mathrm{mmol})$ was added to a suspension of pyridinium chlorochromate $(4.20 \mathrm{~g}, 19.3 \mathrm{mmol})$ in dry $\mathrm{CH}_{2} \mathrm{Cl}_{2}\left(105 \mathrm{~cm}^{3}\right)$ at room temperature. After $3 \mathrm{~h}$, dry ether $\left(100 \mathrm{~cm}^{3}\right)$ was added and the mixture was filtered through a three layer column containing Celite, silica gel and charcoal and then concentrated. The oil was purified by column chromatography (hexane: ethyl acetate, $5: 1 \mathrm{v} / \mathrm{v}$ ) to afford ketone 5 (3.11 g, 93\% yield). $[\alpha]_{\mathrm{D}}{ }^{25}-10.8\left(c 1.30, \mathrm{CHCl}_{3}\right)$, Lit. ${ }^{7}:[\alpha]_{\mathrm{D}}{ }^{25}-10.5$ (c 36.8, $\left.\mathrm{CHCl}_{3}\right)$ ]; IR v max. $/ \mathrm{cm}^{-1} 3088$, 3063, 3030, 2953, 2926, 2869, 1708, 1454, 1099 (film);. ${ }^{1} \mathrm{H}$ NMR $\left(200 \mathrm{MHz}, \mathrm{CDCl}_{3}\right) \delta 1.00(\mathrm{~d}, J 6.3 \mathrm{~Hz}, 3 \mathrm{H}), 1.01$ 
$(\mathrm{d}, J 6.9 \mathrm{~Hz}, 3 \mathrm{H}), 1.32-1.44(\mathrm{~m}, 1 \mathrm{H}), 1.70-1.78(\mathrm{~m}, 1 \mathrm{H}), 1.79-$ $1.89(\mathrm{~m}, 2 \mathrm{H}), 1.97(\mathrm{dd}, J 1.2$ and $12.9 \mathrm{~Hz}, 1 \mathrm{H}), 2.01-2.08(\mathrm{~m}$, $1 \mathrm{H}), 2.16$ (hept, $J 6.7 \mathrm{~Hz}, 1 \mathrm{H}), 2.31-2.37$ (m, 2H), 3.38 (dd, $J$ 6.0 and $9.1 \mathrm{~Hz}, 1 \mathrm{H}), 3.48(\mathrm{dd}, J 5.4$ and $9.1 \mathrm{~Hz}, 1 \mathrm{H}), 4.46(\mathrm{~d}$, $J 15.2 \mathrm{~Hz}, 1 \mathrm{H}), 4.49$ (d, $J 15.2 \mathrm{~Hz}, 1 \mathrm{H}), 7.24-7.27$ (m, 5H); ${ }^{13} \mathrm{C} \mathrm{NMR}\left(50 \mathrm{MHz}, \mathrm{CDCl}_{3}\right) \delta 15.5,22.3,29.5,32.7,34.1$, 35.5, 51.0, 52.2, 72.9, 73.0, 127.5 (3C), 128.3 (2C), 138.7, 212.0; GC-MS (70 eV) m/z $260\left(\mathrm{M}^{+}, 1.2\right) ; 202$ (21.4); 169 (24.9); 139 (25.0); 112 (80.8); 91 (100); 69.15 (53,2); 55 (61.2).

(+)-(3R,6S)-3-Methyl-6-[(1'S)-1'-methyl-2-benzyloxymethyl]-E-caprolactone (6)

Ketone 5 (3.07 g, $11.8 \mathrm{mmol})$ was added to a stirred solution of $m$-CPBA $(40 \%, 6.04 \mathrm{~g}, 15.3 \mathrm{mmol})$ in $\mathrm{CH}_{2} \mathrm{Cl}_{2}$ $\left(80 \mathrm{~cm}^{3}\right)$ containing a suspension of $\mathrm{NaHCO}_{3}(6.00 \mathrm{~g}, 71$ $\mathrm{mmol}$ ). After stirring for $20 \mathrm{~h}$ at room temperature, aqueous $\mathrm{KI}\left(40 \%, 24 \mathrm{~cm}^{3}\right)$ and aqueous $\mathrm{NaHSO}_{3}\left(40 \%, 24 \mathrm{~cm}^{3}\right)$ were added to reduce excess oxidant. The resulting two phase system was stirred for $10 \mathrm{~min}$. After separation, the organic phase was washed with brine $\left(20 \mathrm{~cm}^{3}\right)$, dried over $\mathrm{Na}_{2} \mathrm{SO}_{4}$, concentrated and the residue was purified by column chromatography (hexane: ethyl acetate, 5:1v/v) to afford lactone $6(2.45 \mathrm{~g}, 75 \%$ yield $) .[\alpha]_{\mathrm{D}}{ }^{25}+2.5(c$ 0.85, $\left.\mathrm{CHCl}_{3}\right) ; \mathrm{IR} v_{\text {max. }} / \mathrm{cm}^{-1}$ 3033, 2959, 2928, 2871, 1729, 1454, 1102 (film); ${ }^{1} \mathrm{H}$ NMR (400 MHz, $\mathrm{CDCl}_{3}$ ) $\delta 0.96(\mathrm{~d}, J$ $7.0 \mathrm{~Hz}, 3 \mathrm{H}), 1.04$ (d, J 6.7 Hz, 3H), 1.29-1.38 (m, 1H), 1.66$1.78(\mathrm{~m}, 2 \mathrm{H}), 1.78-1.88(\mathrm{~m}, 1 \mathrm{H}), 1.88-1.98(\mathrm{~m}, 2 \mathrm{H}), 2,45(\mathrm{dt}$, $J 1.8$ and $13.4 \mathrm{~Hz}, 1 \mathrm{H}), 2,54$ (dd, $J 11.5$ and $13.4 \mathrm{~Hz}, 1 \mathrm{H}$ ), $3.36(\mathrm{dd}, J 5.3$ and $9.2 \mathrm{~Hz}, 1 \mathrm{H}), 3.49$ (t, $J 9.0 \mathrm{~Hz}, 1 \mathrm{H})$; 4.49 (s, $2 \mathrm{H}), 4.49-4.52(\mathrm{~m}, 1 \mathrm{H}), 7.26-7.35(\mathrm{~m}, 5 \mathrm{H}) ;{ }^{13} \mathrm{C} \mathrm{NMR}(100$ $\left.\mathrm{MHz}, \mathrm{CDCl}_{3}\right) \delta 10.2,24.0,30.3,32.1,37.3,39.4,42.5,71.8$, 73.2, 79.0, 127.7 (3C), 128.4 (2C), 138.3, 175.0; GC-MS $(70 \mathrm{eV}) \mathrm{m} / \mathrm{z}, 276\left(\mathrm{M}^{+}, 3.4\right) ; 230$ (1.2); 170 (1.6); 152 (9.8); 128 (10.1); 11 (71.7); 91 (100); 69 (51.0) 55 (65.5).

\section{(-)-Methyl (3R,6S,7S)-8-benzyloxy-6-hydroxy-3,7-dimethyl- octanoate (7)}

Sulfuric acid (98\%, 5 drops) was added to lactone 6 $(2.30 \mathrm{~g}, 8.3 \mathrm{mmol})$ in $\mathrm{MeOH}\left(25 \mathrm{~cm}^{3}\right)$, and the mixture was refluxed for $30 \mathrm{~min}$. After cooling, saturated aqueous $\mathrm{NaHCO}_{3}\left(10 \mathrm{~cm}^{3}\right)$ was added, most of the $\mathrm{MeOH}$ was evaporated and the residue was extracted with ether $(3 \mathrm{x}$ $\left.25 \mathrm{~cm}^{3}\right)$. The organic phase was washed with brine $(20$ $\mathrm{cm}^{3}$ ), dried over $\mathrm{Na}_{2} \mathrm{SO}_{4}$, concentrated and the residue was purified by column chromatography (hexane: ethyl acetate, $5: 2 \mathrm{v} / \mathrm{v})$ to afford the desired hydroxy-ester $7(2.39 \mathrm{~g}, 93 \%$ yield). $[\alpha]_{\mathrm{D}}{ }^{25}-1.70\left(\right.$ c $\left.2.88, \mathrm{CHCl}_{3}\right)$, Lit. ${ }^{7}:[\alpha]_{\mathrm{D}}{ }^{25}-1.10$ (c 3.6, $\mathrm{CHCl}_{3}$ )]; IR v max. $/ \mathrm{cm}^{-1} 3491,3063,3030,2954$, 2672, 1725 1454, 1099, 1010 (film); ${ }^{1} \mathrm{H}$ NMR (400 MHz,
$\left.\mathrm{CDCl}_{3}\right) \delta 0.92(\mathrm{~d}, J 7.0 \mathrm{~Hz}, 3 \mathrm{H}), 0.94(\mathrm{~d}, J 6.6 \mathrm{~Hz}, 3 \mathrm{H})$, $1.12-1.23(\mathrm{~m}, 1 \mathrm{H}), 1.38-1.47$ (m, 2H), 1.48-1.58 (m, 1H), 1.84-2.03 (m, 2H), 2.12 (dd, $J 8.2$ and $14.8 \mathrm{~Hz}, 1 \mathrm{H}), 2.32$ (dd, $J 5.9$ and $14.8 \mathrm{~Hz}, 1 \mathrm{H}), 2.57$ (br s, 1H), 3.52 (d, J 6.0 $\mathrm{Hz}, 1 \mathrm{H}), 3.52(\mathrm{~d}, J 5.0 \mathrm{~Hz}, 1 \mathrm{H}), 3.65(\mathrm{~s}, 3 \mathrm{H}), 3.72(\mathrm{td}, J 2.5$ and $6.7 \mathrm{~Hz}, 1 \mathrm{H}), 4.48(\mathrm{~d}, J 11.7 \mathrm{~Hz}, 1 \mathrm{H}), 4.52(\mathrm{~d}, J 11.7 \mathrm{~Hz}$, 1H), 7.26-7.37 (m, 5H); ${ }^{13} \mathrm{C} \mathrm{NMR}\left(100 \mathrm{MHz}, \mathrm{CDCl}_{3}\right) \delta$ 10.6, 19.7, 30.4, 31.2, 33.2, 37.7, 41.4, 51.3, 73.3, 74.1, 74.7, 127.5 (2C), 127.6, 128.4 (2C), 137.9, 173.6; GC-MS $(70 \mathrm{eV}) \mathrm{m} / \mathrm{z} 276$ (1.8), 230 (0.8), 184 (0.8), 152 (6.6), 127 (6.6), 110 (41.0), 91 (100), 81 (20.4), 69 (30.8), 55 (37.0).

(+)-Methyl (3R,6S, 7S)-8-benzyloxy-3,7-dimethyl-6-ptoluene-sulfonyloxyoctanoate $(8)$

Tosyl chloride $(2.61 \mathrm{~g}, 13.6 \mathrm{mmol})$ was added in small portions $(1 \mathrm{~h})$ to ester $7(2.10 \mathrm{~g}, 6.8 \mathrm{mmol})$ in dry pyridine (1.61 g, $20.4 \mathrm{mmol})$, DMAP (20 mg) and chloroform $\left(10 \mathrm{~cm}^{3}\right)$, with magnetic stirring at $0{ }^{\circ} \mathrm{C}$. After $12 \mathrm{~h}$, ether $\left(15 \mathrm{~cm}^{3}\right)$ was added and the solution was thoroughly washed with aqueous $\mathrm{HCl}\left(10 \%, 10 \mathrm{~cm}^{3}\right)$ and saturated solution of $\mathrm{NaHCO}_{3}\left(10 \mathrm{~cm}^{3}\right)$. The organic layer was dried over $\mathrm{Na}_{2} \mathrm{SO}_{4}$, concentrated and the oil obtained was purified by column chromatography (hexane:ethyl acetate, $9: 1 \mathrm{v} / \mathrm{v}$ ) to afford compound $\mathbf{8}$ as a colorless oil ( $2.26 \mathrm{~g}, 72 \%$ yield). $[\alpha]_{\mathrm{D}}{ }^{25}+5.58\left(c 1.54, \mathrm{CHCl}_{3}\right) ; \mathrm{IR} v_{\max } / \mathrm{cm}^{-1} 3063,3030$, 2955, 2873, 1736, 1362, 1175, 903 (film); ${ }^{1} \mathrm{H}$ NMR (200 $\left.\mathrm{MHz}, \mathrm{CDCl}_{3}\right) \delta 0.86(\mathrm{~d}, J 6.6 \mathrm{~Hz}, 3 \mathrm{H}), 0.89(\mathrm{~d}, J 7.0 \mathrm{~Hz}, 3$ $\mathrm{H}), 1.01-1.36(\mathrm{~m}, 2 \mathrm{H}), 1.58-1.70(\mathrm{~m}, 2 \mathrm{H}), 1.78-1.96(\mathrm{~m}$, $1 \mathrm{H}), 1.97-2.10$ (m, $2 \mathrm{H}), 2.19$ (dd, J 6.2 and $15.0 \mathrm{~Hz}, 1 \mathrm{H})$, 2.42 (s, $3 \mathrm{H}), 3.25$ (d, J 6.6 Hz, $2 \mathrm{H}), 3.64$ (s, 3H), 4.31 (d, J $11.8 \mathrm{~Hz}, 1 \mathrm{H}), 4.41(\mathrm{~d}, J 11.8 \mathrm{~Hz}, 1 \mathrm{H}), 4.79(\mathrm{td}, J 3.0$ and 7.0 $\mathrm{Hz}, 1 \mathrm{H}), 7.26-7.35(\mathrm{~m} 7 \mathrm{H}), 7.77(\mathrm{~d}, J 8.0 \mathrm{~Hz}, 2 \mathrm{H}) ;{ }^{13} \mathrm{C}$ $\mathrm{NMR}\left(50 \mathrm{MHz}, \mathrm{CDCl}_{3}\right) \delta 11.0,19.5,21.6,29.4,30.1,32.0$, 36.6, 41.3, 51.4, 71.7, 73.0, 84.5, 127.6 (3C), 127.7 (2C), 128.4 (2C), 129.7 (2C), 134.7, 138.4, 144.4, 173.3.

\section{(+)-(3R,7R)-8-Benzyloxy-3,7-dimethyloctan-1-ol (9)}

Tosylate 8 (2.20 g, $4.8 \mathrm{mmol})$ was dissolved in dry ether $\left(200 \mathrm{~cm}^{3}\right)$ and then $\mathrm{LiAlH}_{4}(1.63 \mathrm{~g}, 42.8 \mathrm{mmol})$ was added. The mixture was stirred at room temperature for $3 \mathrm{~h}$. Excess hydride was destroyed by addition of water (2.0 $\left.\mathrm{cm}^{3}\right)$, aqueous $\mathrm{NaOH}\left(15 \%, 2.0 \mathrm{~cm}^{3}\right)$ and water again $(8.0$ $\mathrm{cm}^{3}$ ). The suspension was filtered through Celite, dried over $\mathrm{Na}_{2} \mathrm{SO}_{4}$ and concentrated. The product obtained was purified by column chromatography (hexane: ethyl acetate, $5: 1 \mathrm{v} / \mathrm{v})$ affording alcohol $9(0.84 \mathrm{~g}, 67 \%$ yield $) .[\alpha]_{\mathrm{D}}{ }^{25}+$ $3.0\left(\right.$ c 1.70, $\left.\mathrm{CHCl}_{3}\right)$, Lit. $^{7}:[\alpha]_{\mathrm{D}}{ }^{25}+3.57\left(\right.$ c 2.8, $\left.\mathrm{CHCl}_{3}\right)$; IR $v_{\text {max. }} / \mathrm{cm}^{-1} 3374,3064,3030,2953,2928,1454,1100$ (film); ${ }^{1} \mathrm{H} \mathrm{NMR}\left(200 \mathrm{MHz}, \mathrm{CDCl}_{3}\right) \delta 0.89(\mathrm{~d}, J 6.8 \mathrm{~Hz}$, $3 \mathrm{H}), 0.92$ (d, J 7.0 Hz, 3H), 1.00-1.80 (m 11H), 3.24 (dd, $J$ 
6.6 and $9.0 \mathrm{~Hz}, 1 \mathrm{H}), 3.32(\mathrm{dd}, J 6.4$ and $9.0 \mathrm{~Hz}, 1 \mathrm{H}), 3.67$ (ddd, $J 2.2,6.4$ and $8.8 \mathrm{~Hz}, 2 \mathrm{H}), 4.50(\mathrm{~s}, 2 \mathrm{H}) ; 7.26-7.35(\mathrm{~m}$, $5 \mathrm{H}) ;{ }^{13} \mathrm{C} \mathrm{NMR}\left(50 \mathrm{MHz}, \mathrm{CDCl}_{3}\right) \delta 17.1,19.6,24.2,29.4$, $33.4,33.9,37.3,40.0,61.2,73.0,76.0,127.4,127.5(2 \mathrm{C})$, 128.3 (2C), 138.8; GC-MS (70 eV) $\mathrm{m} / \mathrm{z} 264\left(\mathrm{M}^{+}, 3.0\right), 155$ (2.0), 107 (34.0), 91 (100), 69 (26.4), 55 (43.2).

(+)-(3R,7R)-8-Benzyloxy-3,7-dimethyloctyl-ptoluenesulfonylate (10)

Tosyl chloride $(1.17 \mathrm{~g}, 6.0 \mathrm{mmol})$ was added in small portions $(1 \mathrm{~h})$ to alcohol $9(0.80 \mathrm{~g}, 3.0 \mathrm{mmol})$ in dry pyridine $\left(0.8 \mathrm{~cm}^{3}, 9.0 \mathrm{mmol}\right)$, DMAP $(10 \mathrm{mg})$ and chloroform (10 $\left.\mathrm{cm}^{3}\right)$, with magnetic stirring at $0^{\circ} \mathrm{C}$. After $12 \mathrm{~h}$, ether $\left(10 \mathrm{~cm}^{3}\right)$ was added and the solution was thoroughly washed with aqueous $\mathrm{HCl}(10 \%)\left(5 \mathrm{~cm}^{3}\right)$, saturated solution of $\mathrm{NaHCO}_{3}$ $\left(5 \mathrm{~cm}^{3}\right)$, dried, concentrated and the oil obtained was purified by column chromatography (hexane: ethyl acetate, 9:1) to afford the tosylate $\mathbf{1 0}$ as a colorless oil $(1.19 \mathrm{~g}, 93 \%)$. $[\alpha]_{\mathrm{D}}{ }^{25}$ + 1.89 ( $\left.c 7.4, \mathrm{CH}_{2} \mathrm{Cl}_{2}\right)$; Elemental analysis: Found: C, 68.83; $\mathrm{H}, 8.38$; S, 7.93; Calc. for $\mathrm{C}_{24} \mathrm{H}_{34} \mathrm{O}_{4} \mathrm{~S}: \mathrm{C}, 68.87$; H, 8.19; S, $7.66 \%$; IR v max. $/ \mathrm{cm}^{-1} 3063,3031,2956,2928,2857,1454$, 1362, 1177, 944 (film); ${ }^{1} \mathrm{H}$ NMR (400 MHz, $\left.\mathrm{CDCl}_{3}\right) \delta 0.79$ (d, J $6.5 \mathrm{~Hz}, 3 \mathrm{H}), 0.90(\mathrm{~d}, J 6.7 \mathrm{~Hz}, 3 \mathrm{H}), 1.02-1.10(\mathrm{~m}, 2 \mathrm{H})$, $1.10-1.27$ (m, 3H), 1.30-1.46 (m, 2H), 1.48-1.54 (m, 1H), 1.61$1.75(\mathrm{~m}, 2 \mathrm{H}), 2.44(\mathrm{~s}, 3 \mathrm{H}), 3.23(\mathrm{dd}, J 6.6$ and $9.0 \mathrm{~Hz}, 1 \mathrm{H}), 3.29$ (dd, $J 6.2$ and $9.2 \mathrm{~Hz}, 1 \mathrm{H}), 4.05(\mathrm{dt}, J 2.7$ and $6.4 \mathrm{~Hz}, 2 \mathrm{H}), 4.48$ $(\mathrm{d}, J 12.2 \mathrm{~Hz}, 1 \mathrm{H}), 4.51(\mathrm{~d}, J 12.2 \mathrm{~Hz}, 1 \mathrm{H}), 7.25-7.34(\mathrm{~m}, 7 \mathrm{H})$, $7.79(\mathrm{~d}, J 8.3 \mathrm{~Hz}, 2 \mathrm{H}) ;{ }^{13} \mathrm{C} \mathrm{NMR}\left(100 \mathrm{MHz}, \mathrm{CDCl}_{3}\right) \delta 17.0$, 19.0, 21.6, 24.0, 29.1, 33.4, 33.7, 35.7, 36.8, 69.0, 72.9, 75.9, 127.4, 127.5 (2C), $127.8(2 \mathrm{C}), 128.3$ (2C), $129.8(2 \mathrm{C}), 133.1$, 138.7, 144.6; GC-MS (70 eV) m/z 246 (0.8), 155 (2.4), 137 (7.3), 107 (9.5), 91 (100), 69 (29.6), 55 (52.4).

\section{(-)-(2R,6S)-1-Benzyloxy-2,6-dimethyltetradecane (11)}

Magnesium turnings (0.320 g; $13.2 \mathrm{mmol})$ were added to a solution of 1-bromohexane $(2.18 \mathrm{~g}, 13.2 \mathrm{mmol})$ in dry ether $\left(55 \mathrm{~cm}^{3}\right)$ at r. t.. After $6 \mathrm{~h}$, the solution containing the Grignard reagent was added dropwise at $-78{ }^{\circ} \mathrm{C}$ to a previously prepared solution of tosilate $\mathbf{1 0}(1.10 \mathrm{~g}, 2.6$ $\mathrm{mmol})$ in dry THF $\left(55 \mathrm{~cm}^{3}\right)$. Then, a solution of $\mathrm{Li}_{2} \mathrm{CuCl}_{4}$ in THF $0.1 \mathrm{~mol} \mathrm{dm}^{-3}\left(0.6 \mathrm{~cm}^{3}\right)$ was added to the mixture. The reaction temperature was raised slowly until r. t. ( $2 \mathrm{~h})$ and stirred for $10 \mathrm{~h}$. The reaction was quenched with saturated solution of $\mathrm{NH}_{4} \mathrm{Cl}\left(40 \mathrm{~cm}^{3}\right)$ and then extracted with ether $\left(3 \times 30 \mathrm{~cm}^{3}\right)$. The organic layer was washed with saturated $\mathrm{NaHCO}_{3}$ solution $\left(50 \mathrm{~cm}^{3}\right)$ and brine $\left(50 \mathrm{~cm}^{3}\right)$, dried over $\mathrm{MgSO}_{4}$, filtered, and evaporated in vacuo. The residue was purified by column chromatography in silica gel (hexane:ethyl acetate, 95:5v/v) affording compound 11 $(0.72 \mathrm{~g}, 90 \%$ yield $) .[\alpha]_{\mathrm{D}}{ }^{25}-0.17\left(c 3.4, \mathrm{CH}_{2} \mathrm{Cl}_{2}\right)$; Elemental analysis: Found: C, 82.90; H, 11.85. Calc. for $\mathrm{C}_{23} \mathrm{H}_{40} \mathrm{O}$ : C, 83.07; H, 12.12; IR v max. $/ \mathrm{cm}^{-1} 3064,3030,2954,2925$, 2854, 1457, 1376, 1100, 733 (film); ${ }^{1} \mathrm{H}$ NMR (200 MHz, $\left.\mathrm{CDCl}_{3}\right) \delta 0.83(\mathrm{~d}, J 6.2 \mathrm{~Hz}, 3 \mathrm{H}), 0.88(\mathrm{t}, J 6.5 \mathrm{~Hz}, 3 \mathrm{H}), 0.92$ $(\mathrm{d}, J 6.8 \mathrm{~Hz}, 3 \mathrm{H}), 1.26(\mathrm{~m}, 21 \mathrm{H}), 1.60-1.90(\mathrm{~m}, 1 \mathrm{H}), 3.23(\mathrm{dd}$, $J 6.6$ and $9.0 \mathrm{~Hz}, 1 \mathrm{H}), 3.33(\mathrm{dd}, J 6.2$ and $9.0 \mathrm{~Hz}, 1 \mathrm{H}), 4.50(\mathrm{~s}$, $2 \mathrm{H}), 7.18-7.30(\mathrm{~m}, 5 \mathrm{H}) ;{ }^{13} \mathrm{C} \mathrm{NMR}\left(50 \mathrm{MHz}, \mathrm{CDCl}_{3}\right) \delta 14.1$, 17.2, 19.7, 22.7, 24.4, 27.1, 29.4, 29.7, 30.1, 32.0, 32.7, 33.5, 34.0, 37.2, 37.3, 73.0, 76.1, 127.4, 127.5 (2C), 128.3 (2C), 138.9; GC-MS (70 eV) m/z 246 (0.8), 155 (2.4), 137 (7.3), 107 (9.5), 91 (100), 69 (29.6), 55 (52.4).

\section{(+)-(2R,6S)-2,6-Dimethyltetradecan-1-ol(12)}

A mixture of benzyl ether $\mathbf{1 1}(0.70 \mathrm{~g}, 2.1 \mathrm{mmol})$ and $10 \%$ $\mathrm{Pd} / \mathrm{C}(0.10 \mathrm{~g})$ in ethanol $\left(5 \mathrm{~cm}^{3}\right)$ was hydrogenated at room temperature under hydrogen atmosphere for $12 \mathrm{~h}$. The mixture was filtered through Celite, and the filtrate evaporated at reduced pressure to afford alcohol $12(0.37 \mathrm{~g}, 73 \%$ yield $)$. $[\alpha]_{\mathrm{D}}{ }^{25}+8.56\left(c\right.$ 1.46, $\left.\mathrm{CH}_{2} \mathrm{Cl}_{2}\right)$; Elemental analysis: Found: C, 79.16; H, 14.36; Calc. for $\mathrm{C}_{16} \mathrm{H}_{34} \mathrm{O}$ : C, 79.27; H, 14.14; IR $v_{\text {max }} / \mathrm{cm}^{-1} 3345,2955,2924,2854,1463,1377,1034$, 722 (film); ${ }^{1} \mathrm{H}$ NMR $\left(200 \mathrm{MHz}, \mathrm{CDCl}_{3}\right) \delta 0.84(\mathrm{~d}, J 6.2 \mathrm{~Hz}$, $3 \mathrm{H}), 0.88(\mathrm{t}, J 6.5 \mathrm{~Hz}, 3 \mathrm{H}), 0.92(\mathrm{~d}, J 6.8 \mathrm{~Hz}, 3 \mathrm{H}), 1.00-1.44(\mathrm{~m}$, $22 \mathrm{H}), 1.50-1.72(\mathrm{~m}, 1 \mathrm{H}), 3.46(\mathrm{dq}, J 10.2$ and $5.8 \mathrm{~Hz}, 2 \mathrm{H}) ;{ }^{13} \mathrm{C}$ NMR (50 MHz, $\left.\mathrm{CDCl}_{3}\right) \delta 14.1,16.6,19.7,22.7,24.4,27.1$, 29.4, 29.7, 30.1 , 32.0, 32.8, 33.5, 35.8, 37.2, 37.3, 68.5; GCMS (70 eV) m/z 154 (0.7), 140 (1.9), 126 (4.1), 112 (4.0), 111 (19.5), 99 (1.8), 98 (5.1), 85 (11.9), 84 (6.3), 83 (14.1), 71 (29.3), 70 (24.4), 69 (66.6), 57 (100), 56 (56.2), 55 (98.5).

\section{(-)-(2R,6S)-2,6-Dimethyltetradecan-1-al (13)}

Alcohol $12(0.10 \mathrm{~g}, 0.4 \mathrm{mmol})$ was added to a suspension of PCC $(0.14 \mathrm{~g}, 0.6 \mathrm{mmol})$ in dry $\mathrm{CH}_{2} \mathrm{Cl}_{2}$ $\left(5.0 \mathrm{~cm}^{3}\right)$. After $3 \mathrm{~h}$, dry ether $\left(5.0 \mathrm{~cm}^{3}\right)$ was added and the mixture was filtered through a three-layer column containing Celite, silica gel and charcoal and then concentrated. The residue was purified by column chromatography (pentane:ether, 3:1v/v) to afford aldehyde $13(0.080 \mathrm{~g}, 81 \%$ yield). $[\alpha]_{\mathrm{D}}{ }^{25}-11.85\left(c 1.3, \mathrm{CH}_{2} \mathrm{Cl}_{2}\right.$ ); Elemental analysis: Found: C, 79.79; H, 13.41; Calc. for $\mathrm{C}_{16} \mathrm{H}_{32} \mathrm{O}: \mathrm{C}, 79.93$; $\mathrm{H}$, 13.42; IR $v_{\text {max. }} / \mathrm{cm}^{-1} 2958,2928,2855,2696,1462,1376$, 1237, 1181, 939, 810, 722 (film); ${ }^{1} \mathrm{HNMR}\left(200 \mathrm{MHz}, \mathrm{CDCl}_{3}\right)$ $\delta 0.84(\mathrm{~d}, J 6.2 \mathrm{~Hz}, 3 \mathrm{H}), 0.88(\mathrm{t}, J 6.2 \mathrm{~Hz}, 3 \mathrm{H}), 1.09(\mathrm{~d}, J 7.0 \mathrm{~Hz}$, $3 \mathrm{H}), 1.00-1.50(\mathrm{~m}, 19 \mathrm{H}), 1.50-1.85(\mathrm{~m}, 2 \mathrm{H}), 2.34$ (dsext, $J 2.0$ and $7.0 \mathrm{~Hz}, 1 \mathrm{H}), 9.62(\mathrm{~d}, J 2.0 \mathrm{~Hz}, 1 \mathrm{H}) ;{ }^{13} \mathrm{C} \mathrm{NMR}(50 \mathrm{MHz}$, $\left.\mathrm{CDCl}_{3}\right) \delta 13.3,14.1,19.6,22.7,24.4,27.1,29.4,29.7,30.0$, 30.8, 31.9, 32.6, 37.0 (2C), 46.4, 205.4. GC-MS; $(70 \mathrm{eV}) \mathrm{m} / \mathrm{z}$ 183 (0.6), 127 (1.0), $110(1.6), 109$ (4.3), 95 (2.4), 85 (4.8), 83 (4.0), 81 (7.1), 71 (13.9), 69 (15.6), 68 (4.3), 67 (8.6), 59 (4.2), 58 (100), 57 (49.4), 56 (14.9), 55 (47.6). 
(+)-(2R,3R,7S)-3,7-Dimethylpentadecan-2-ol (1)

A solution of aldehyde $13(0.040 \mathrm{~g}, 0.17 \mathrm{mmol})$ in THF $\left(1.5 \mathrm{~cm}^{3}\right)$ was cooled at $-100{ }^{\circ} \mathrm{C}$ under nitrogen. To this stirred mixture was added dropwise a solution of $\mathrm{MeMgBr}$ ( $1 \mathrm{~mol} \mathrm{dm}-3$ in ether, $0.20 \mathrm{~cm}^{3}, 0.20 \mathrm{mmol}$ ), and the reaction temperature was raised slowly to r.t. This mixture was stirred at r. t. for $12 \mathrm{~h}$. A saturated solution of $\mathrm{NH}_{4} \mathrm{Cl}\left(1.0 \mathrm{~cm}^{3}\right)$ was added and the reaction mixture was extracted with ether ( 3 $\left.\mathrm{x} 3 \mathrm{~cm}^{3}\right)$. The organic layer was washed with brine $\left(5 \mathrm{~cm}^{3}\right)$, dried over $\mathrm{MgSO}_{4}$, filtered, and evaporated in vacuo. The residue was purified by column chromatography (hexane: ethyl acetate, $9: 1 \mathrm{v} / \mathrm{v})$ to afford a $4: 1$ mixture of $(2 R, 3 R, 7 S)$ and $(2 S, 3 R, 7 S)-1$ respectively $(0.029 \mathrm{~g}, 69 \%$ yield). The major product was then purified by column chromatography and obtained as a colorless oil, purity $>96.6 \%$ by chiral GC. $[\alpha]_{\mathrm{D}}{ }^{25}+12.86\left(c 0.14\right.$, hexane), Lit. $^{6}:[\alpha]_{\mathrm{D}}{ }^{25}+13.04$ (neat); Elemental analysis: Found: C, 79.60; H, 14.32; Calc. for
$\mathrm{C}_{17} \mathrm{H}_{36} \mathrm{O}: \mathrm{C}, 79.61 ; \mathrm{H}, 14.15$; IR $v_{\text {max. }} / \mathrm{cm}^{-1} 3345,2957$, 2924, 2854, 1462, 1376, 1097, 1001, 925, 888, 721(film); ${ }^{1} \mathrm{HNMR}\left(400 \mathrm{MHz}, \mathrm{CDCl}_{3}\right) \delta 0.84(\mathrm{~d}, J 6.6 \mathrm{~Hz}, 3 \mathrm{H}), 0.88(\mathrm{t}$, $J 6.3 \mathrm{~Hz} ; 3 \mathrm{H}), 0.89$ (d, J 5.4 Hz, 3H), 1.15 (d, J $6.3 \mathrm{~Hz}, 3 \mathrm{H})$, $1.15-1.50(\mathrm{~m}, 22 \mathrm{H}), 3.70(\mathrm{dq}, J 4.5$ and $6.4 \mathrm{~Hz}, 2 \mathrm{H}) ;{ }^{13} \mathrm{C}$ NMR (50 MHz, $\left.\mathrm{CDCl}_{3}\right) \delta 14.1,19.6,20.3,22.7,24.7,27.1$, 29.4, 29.7, 30.0, 31.9, 32.7, 32.9, 37.2, 37.3, 39.8, 71.5; GCMS (70 eV) m/z 212 (1.0), 183 (0.8), 154 (1.3), 140 (4.1), 125 (2.2), 113 (2.1), 112 (2.6), 111 (3.4), 99 (4.3), 97 (9.2), 85 (12.8), 83 (8.2), 72 (11.6), 71 (22.0), 70 (14.0), 69 (22.2), 57 (100), 56 (52.6), 55 (81.8).

\section{Results and Discussion}

The synthetic route used for the synthesis of $(2 R, 3 R, 7 S)$ diprionol is shown in Scheme 1. The stereogenic center at C3 of diprionol was established through a stereoselective hydroboration of (-)-isopulegol (2), with a diastereoisomeric

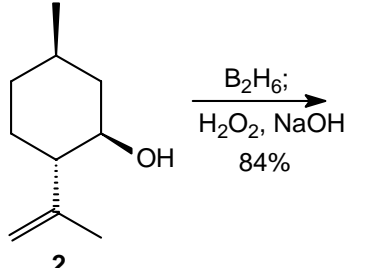

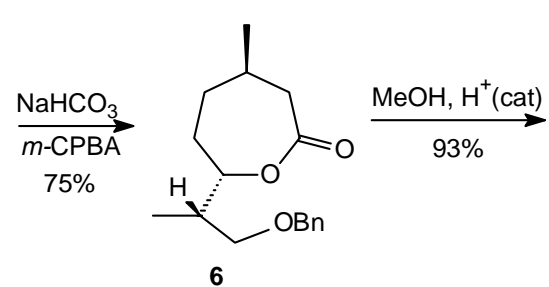

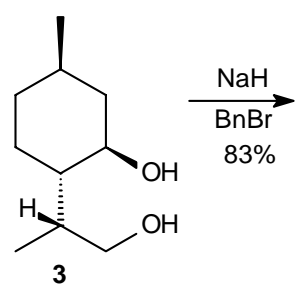<smiles>C[C@H](COCc1ccccc1)[C@H](C)COc1ccccc1</smiles><smiles>CC1CCC(C(C)COCc2ccccc2)C(=O)C1</smiles><smiles>COC(=O)CC(C)CC[C@@H](O)[C@@H](C)COc1ccccc1</smiles><smiles>COC(=O)CC(C)CC[C@@H](O)[C@H](C)COCc1ccccc1</smiles>

8<smiles>CC(CCO)CCC[C@H](C)COCc1ccccc1</smiles><smiles>CC(CC[Se-])CCC[C@H](C)COCc1ccccc1</smiles>

10

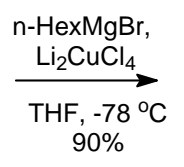<smiles>CCCCCCCCC(C)CCCC(C)C[C@H](C)Cc1ccccc1</smiles>

11

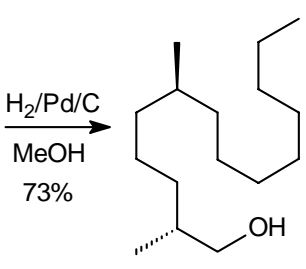

12

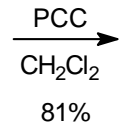<smiles>CCCCCCCCC(C)CCC[C@H](C)C=O</smiles>

13

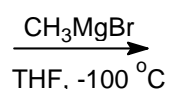

$69 \%, d r=4: 1$<smiles>CCCCCCCC[C@@H](C)CCC[C@H](C)[C@H](C)O</smiles>

Scheme 1. Synthetic route for the preparation of $(2 R, 3 R, 7 S)$-diprionol (1) 
ratio of 7:1. The two stereoisomers of diol $\mathbf{3}$ formed were readily separated by flash column chromatography, followed by recrystallization, affording pure $(1 R, 3 R, 4 S, 8 R)$-diol 3 in $84 \%$ yield. Selective protection of the primary alcohol with benzyl bromide furnished alcohol 4 (83\% yield), which was oxidized with PCC to produce ketone 5 in $93 \%$ yield. BayerVilliger oxidation of this ketone with $m$-CPBA gave lactone 6 in $75 \%$ yield. Methanolysis of this lactone furnished hydroxyester 7 in $93 \%$ yield. To remove the oxygen function at C-3, the tosylate $\mathbf{8}$ was prepared in $72 \%$ yield. Reduction of both tosylate and methyl ester with $\mathrm{LiAlH}_{4}$ gave alcohol 9 in $67 \%$ yield. In this step, we initially obtained a 4:1 mixture of the desired product $\mathbf{9}$ and an olefin resulting from the elimination of the tosyl group. To overcome this problem, the reaction mixture was diluted two times.

Alcohol 9 was converted into its corresponding tosylate 10 (93\% yield) and then coupled with $n$-hexyl magnesium bromide, using $\mathrm{Li}_{2} \mathrm{CuCl}_{4}$ as a catalyst ${ }^{8}$, affording $\mathbf{1 1}$ in $90 \%$ yield. Catalytic hydrogenation of the benzyl group furnished alcohol 12 in 73\% yield. Oxidation of this alcohol with PCC supplied aldehyde $\mathbf{1 3}$ ( $81 \%$ yield), which showed high optical purity by chiral GC. The stability of this aldehyde under basic conditions was also investigated by chiral GC. In dichloromethane solution, in the presence of DMAP at r.t. only after one week we started to detect some epimerization product $(<5 \%)$. However, in the presence of an ethanolic solution of $\mathrm{NaOH}$ at r.t., we observed complete epimerization after $48 \mathrm{~h}$.

The last step of our synthetic route generated the stereogenic center at C-3 of diprionol. According to either Cram's rule or Felkin-Ahn model, the methyl group should add to the $R e$ face of the aldehyde furnishing the $(2 R, 3 R, 7 S)-1$ isomer. However, it is well known from the literature that variations in the reaction conditions may drastically influence the stereoselectivity of the addition.

In order to determine the optimal conditions for the nucleophilic addition to aldehyde 13, $(S)$-2-methylbutanal was employed as a model compound in a systematic study. Organometallic reagents (alkyl lithium, magnesium, cuprate, and zinc) were tested at different temperatures (from 25 to $80^{\circ} \mathrm{C}$ ), and solvent (THF, diethylether and toluene). We also tried the mediation of a Lewis acid $\left(\mathrm{Ti}\left(\mathrm{O}^{i} \mathrm{Pr}\right)_{4}\right)$ with and without a chiral ligand ((2R,3R)-2,3-O-(1-phenylethylidene)-1,1,4,4tetraphenyl-1,2,3,4-butanetetrol $)^{9}$. However, the asymmetric induction was always very low, with a diastereoisomeric ratio (dr), measured by chiral GC, varying from 54:46 to 66:34. The best result $(\mathrm{dr}=71: 29)$ was obtained when diethyl zinc was employed in diethylether at room temperature. Nevertheless, this organometallic reagent can not be used in our synthetic route.

Based on these results, we decided to employ methyl magnesium bromide in THF in the nucleophilic addition to aldehyde 13. When the reaction was carried out at $-78{ }^{\circ} \mathrm{C}$, the diastereoisomeric ratio was $66: 34$ by chiral $\mathrm{GC}$, but at $-100^{\circ} \mathrm{C}$ we obtained a $4: 1$ ratio and $69 \%$ isolated yield, having $(2 R, 3 R, 7 S)-\mathbf{1}$ as the major product. The absolute configuration at $\mathrm{C}-2$ was assigned based on ${ }^{1} \mathrm{H}$ NMR data. According to the literature, the peak corresponding to the methyl group at $\mathrm{C}-3$ for $(2 R, 3 R, 7 S)-1$ is a doublet at $\delta 1.15$ whereas the one corresponding to $(2 S, 3 R, 7 S)-1$ appears at $\delta 1.12$ (Figure 1$)^{6}$.

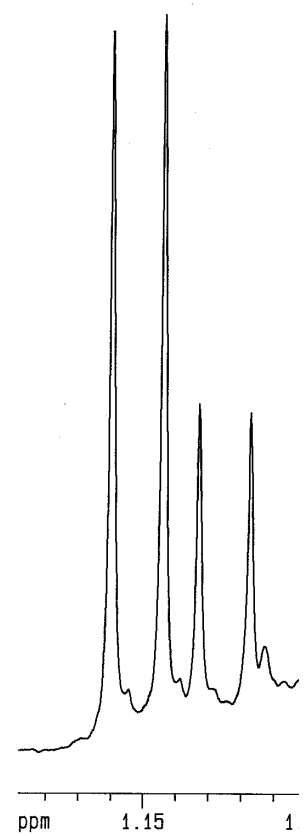

Figure 1. ${ }^{1} \mathrm{H}$ NMR spectroscopy $(400 \mathrm{MHz})$ analysis of a mixture of $(2 R, 3 R, 7 S)$ - and $(2 S, 3 R, 7 S)$-diprionol (1).

Separation of the stereoisomers obtained was quite difficult. After carefull purification by column chromatography, we still observed by chiral GC the presence of the $(2 S, 3 R, 7 S)$ - isomer $(<2.8 \%)$ and a third compound $(<0.6 \%)$, probably the $(2 S, 3 S, 7 S)$ - isomer, which could come from partial epimerization of aldehyde $\mathbf{1 3}$ under the reaction conditions. Thus, the $(2 R, 3 R, 7 S)$ diprionol (1) was obtained in $>96.6 \%$ purity by chiral GC.

Using the same methodology described above, it should be possible to prepare the stereoisomers $(2 S, 3 S, 7 S)$ and/or (2R,3S,7S)-1 starting from (+)-neo-isopulegol, which can be obtained from (-)-isopulegol in 2 steps ${ }^{7}$. The other isomers could also be prepared from commercial available (+)-isopulegol.

\section{Acknowledgments}

The authors are grateful to FAPESP and CNPq, Brazil, for financial support. 


\section{References}

1. Olaifa, J. I.; Kikukawa, T.; Matsumura, F.; Coppel, H. C. Environ. Entomol. 1984, 13, 1274.

2. Anderbrant, O.; Lofqvist, J.; Hogberg, H. E.; Hedenstrom, E.; Baldassari, N.; Baronio, P.; Kolmakova, G.; Lyons, B.; Naito, T.; Odinokov, V.; Simandl, J.; Supatashvili, A.; Tai, A.; Tourianov, R. Entomol. Exp. Appl. 2000, 95, 229.

3. Odinokov, V. N.; Akhmetova, V. R.; Savchenko, R. G. Khim. Prir. Soedin. 1998, 123.

4. (a) Krause, N.; Ebert, S.; Haubrich, A. Liebigs Ann. 1997, 2409. (b) Lundh, M.; Smitt, O.; Hedenstrom, E. Tetrahedron: Asymmetry 1996, 7, 3277. (c) Hedenstrom, E.; Hogberg, H. E. Tetrahedron, 1994, 50,5225 .

5. For reviews, see: (a) Mori, K. The Synthesis of Insect
Pheromones; 1979-1989, In The Total Synthesis of Natural Products, ed. J. ApSimon, vol 9, John Wiley \& Sons, New York; 1981, p. 122-128. (b) Koutek, B.; Streinz, L.; Romanuk, M. Collect. Czech. Chem. Commun. 1998, 63, 899.

6. Högberg, H. E.; Hedenström, E.; Wassgren, A. B.; Hjalmarsson, M.; Bergström, G.; Löfqvist, J.; Norin, T. Tetrahedron 1990, 46, 3007.

7. Ferreira, J. T. B.; Zarbin, P. H. G. Bioorg. Med. Chem. 1996, 4, 381.

8. (a) Burns, D. H.; Miller, J. D.; Chan, H.; Delaney, M. O. J. Am. Chem. Soc. 1997, 119, 2125. (b) Krause, N.; Gerold, A. Angew. Chem. Int. Ed. Engl. 1997, 36, 187.

9. (a) Seebach, D.; Beck, A. K.; Schmidt, B.; Wang, Y. M. Tetrahedron 1994, 50, 4363. (b) Seebach, D.; Plattner, D. A.; Beck, A. K.; Wang, Y. M.; Hunziker, D. Helv. Chim. Acta 1992, 75, 2171.

Received: July 24, 2000 